\title{
Separation of integrated pulse shapes into unique sub-components
}

\author{
Michael Kramer \\ Max-Planck-Institut für Radioastronomie, Bonn, Germany
}

\section{Introduction}

Average pulse profiles are generally separable into several components whereas individual components can be described by a Gaussian shape. One generally distinguishes between core and conal components, although it is yet unclear whether core and conal components originate from the same (Lyne \& Manchester 1988) or a different emission mechanism (Rankin 1983). Investigating this problem, we studied the behaviour of individual components observed in about 200 profiles of 18 pulsars measured at three different frequencies, i.e. at $1.4 \mathrm{GHz}$, $4.75 \mathrm{GHz}$ and $10.55 \mathrm{GHz}$. We unambiguously determined the number, location, width, flux density and spectral index of individual components using a special technique (Kramer et al. 1994). The applied method ensures that only intrinsic features of the pulse profiles are fitted by comparing off-pulse noise statistics and post-fit residuals as a goodness-of-fit estimator. As a first result, we note that all high $\mathrm{S} / \mathrm{N}$ pulsar profiles can be accurately described by using only a small number (usually $\leq 5$ ) of gaussian components, although we also find an example of a seven component pulse shape (B0740-28). Fitted components determined for different observations of the same pulsar were averaged, producing a grand mean profile which is completely noise-free and thus suitable for a reliable flux density measurement of individual components, for an accurate determination of the pulse width to calculate opening angles, $\rho$, of the emission cone, or for use as a template for obtaining pulse arrival times.

\section{Results and conclusions}

Using the above results, published values for pulsar geometries (Lyne \& Manchester 1988, Rankin 1993b), and assuming circular beams, we calculated $\rho$ corresponding to the $10 \%$-intensity width (see Gil et al. 1984, Kramer et al. 1994). In accordance with the results of Rankin (1993a) and Gil et al. (1993), we find that opening angles are generally located on two lines if they are plotted versus period in a log-log diagram. Similar results are also obtained for the opening angles of individual components, although here the scatter is larger, probably due to asymmetries in pulse profiles and remaining uncertainties in the fit results. Generally, the number of components and the frequency development of the pulse profiles can be well described in context of a double cone model (Rankin 1993a). In this model the inner components are either identified with core components or created if the line-of-sight just grazes the inner cone. Accepting this model, components can be unambiguously classified as the core or 


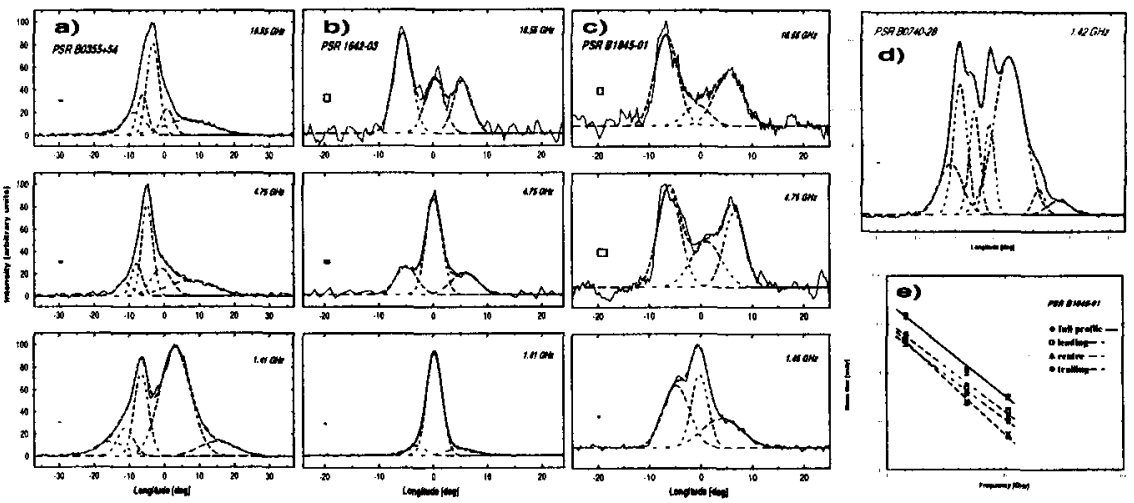

Figure 1. Examples for fit results obtained for a) B0355+54 b) B1642-03 and c) B1845-01. d) B0740-28 exhibits a seven component profile. e) Spectra of individual components for B1845-01.

by designating the corresponding cone (Kramer 1994). We observe that components caused by grazing the inner cone show the same spectral behaviour as core components, i.e. they exhibit a spectrum steeper than those of the outer profile components. Such a steeper spectrum was observed as one of the basic properties of core emission and considered as an indication for a different emission mechanism (Rankin 1983a). The fact that both types of components, i.e. inner cones and cores, exhibit such a phenomenon points towards a geometrical rather than a physical origin of the steep spectrum (i.e. assuming a radius-to-frequency mapping, inner components and the core tend to move out of the line-of-sight at higher frequencies). Therefore, we conclude that there is no fundamental difference in the spectral behaviour of core and conal components.

Acknowledgments. I am indebted to R. Wielebinski for his helpful advice and constant support. I appreciate particular valuable discussions with A. Jessner, and I am grateful to J. Gil and J.H. Seiradakis for their help and our great collaboration. I would also like to thank all participants of the Pulsar-Month, D. Graham for help with the 1992 observations, and V.M. Malofeev, W. Sieber and especially K.M. Xilouris for helpful and stimulating discussions.

\section{References}

Gil J.A., Gronkowski P., Rudnicki W. 1984, A\&A, 132, 312

Gil J.A., Kijak J., Seiradakis J.H. 1993, A\&A, 272, 268

Kramer M., 1994, A\&AS, 107, 527

Kramer M., Wielebinski R., Jessner A., et al., 1994, A\&A, 107, 515

Lyne A.G., Manchester R.N. 1988, MNRAS, 234, 447

Rankin J.M. 1983, ApJ, 274, 333

Rankin J.M. 1993a, ApJ, 405, 285

Rankin J.M. 1993b, ApJS, 85, 145 